\title{
CONCENTRACIÓN NUTRIMENTAL FOLIAR Y CRECIMIENTO DE CHILE SERRANO EN FUNCIÓN DE LA SOLUCIÓN NUTRITIVA Y EL SUSTRATO
}

\section{LEAF NUTRIENT CONCENTRATION AND PLANT GROWTH OF CHILI SERRANO IN RELATION TO NUTRIENT SOLUTION AND SUBSTRATE}

\author{
Elia Cruz-Crespo ${ }^{1 *}$, Álvaro Can-Chulim ${ }^{1}$, Rubén Bugarín-Montoya ${ }^{1}$, Joel Pineda-Pineda ${ }^{2}$, \\ Ricardo Flores-Canales ${ }^{1}$, Porfirio Juárez-López ${ }^{3}$ y Gelacio Alejo-Santiago ${ }^{1}$
}

\begin{abstract}
${ }^{1}$ Unidad Académica de Agricultura, Universidad Autónoma de Nayarit. Km 9 Carr. Tepic-Compostela. 63780, Xalisco, Nayarit. Tel. $3112110128 .{ }^{2}$ Departamento de Suelos, Universidad Autónoma Chapingo. Km 38.5 Carr. México-Texcoco. 56230, Chapingo, Texcoco, Edo. de México. ${ }^{3}$ Facultad de Ciencias Agropecuarias, Universidad Autónoma del Estado de Morelos. Avenida Universidad 1001. 62210, Cuernavaca, Morelos, México.
\end{abstract}

*Autor para correspondencia (ccruzc2006@yahoo.com.mx)

\section{RESUMEN}

El cultivo en sustrato favorece el crecimiento y rendimiento de los cultivos; sin embargo, esto depende de sus características y del requerimiento del cultivo. El objetivo del presente trabajo fue estudiar algunos sustratos de la región de Nayarit, México, formados por tezontle y por la mezcla de tezontle con lombricomposta (bagazo de caña (Saccharum officinarum), hueso de mango (Mangifera indica), estiércol de bovino) regados con la solución nutritiva de Steiner a concentraciones de 25,50 y $75 \%$ (diluciones). Se midió el contenido nutrimental en tejido, crecimiento y rendimiento de chile serrano (Capsicum annuum $\mathrm{L}$.) bajo invernadero. Los tratamientos se formaron con un arreglo factorial $3 \times 2$ que correspondieron a tres diluciones y dos sustratos; se utilizó un diseño experimental completamente al azar con ocho repeticiones. Los sustratos se caracterizaron física y químicamente. Las variables evaluadas fueron diámetro de tallo, número de ramificaciones, biomasa seca de planta, rendimiento de fruto y la concentración de N, P, K, Ca y Mg foliar. Con excepción del rendimiento de fruto, todas las variables se midieron a los 40 y $80 \mathrm{~d}$ después del trasplante. Los resultados mostraron que la dilución a $75 \%$ incrementó el crecimiento, rendimiento de fruto, así como la concentración de $\mathrm{N}$, Ca y Mg en tejido foliar, cuyos valores se ubicaron en el rango de suficiencia, a excepción de P y Ca. El sustrato tezontle/ lombricomposta produjo el mayor crecimiento de la planta, así como la concentración de $\mathrm{P}$ y Mg en el tejido foliar, pero el rendimiento de fruto fue similar al obtenido en el sustrato tezontle.

Palabras clave: Capsicum annuum, substratos, biomasa seca, rendimiento.

\section{SUMMARY}

Substrate culture may promote crop growth and yield; however, this depends on the substrate properties and the crop requirements. In this work we studied some substrates from Nayarit, México, formed by volcanic rock and by the mixture of volcanic rock with a vermicompost (obtained from sugar (Saccharum officinarum) cane bagasse, bone of mango (Mangifera indica), cattle manure), on a serrano crop (Capsicum annuum L.) grown under greenhouse conditions and irrigated with the Steiner nutrient solution at 25,50 and $75 \%$ dilutions. Nutrient concentration in plant tissue, growth and performance of serrano chile were measured. Treatments formed a factorial arrangement $3 \times 2$ corresponding to three dilutions and two substrates, in a randomized experimental design with eight replications. The substrates were physically and chemically characterized. The variables evaluated were: stem diameter, number of branches, plant dry biomass, fruit yield, and leaf concentration of $\mathrm{N}, \mathrm{P}, \mathrm{K}, \mathrm{Ca}$ and $\mathrm{Mg}$. With the exception of fruit yield, all variables were measured at 40 and $80 \mathrm{~d}$ after transplant. The results showed that plant growth, fruit yield, as well as leaf $\mathrm{N}, \mathrm{Ca}$ and $\mathrm{Mg}$ concentration increased in plants growing in the $75 \%$ dilution; and nutrients reached their optimum levels, except $P$ and $\mathrm{Ca}$. The substrate formed by the mixture of volcanic rock and vermicompost also increased plant growth and leaf concentration of $\mathrm{P}$ and $\mathrm{Mg}$, although fruit yield was similar to that obtained in volcanic rock.

Index words: Capsicum annuum, substrates, dry biomass, yield.

\section{INTRODUCCIÓN}

Hoy en día existe creciente necesidad de encontrar técnicas que contribuyan al uso eficiente del agua y nutrientes, lo cual se pretende lograr con el uso de diferentes sustratos inorgánicos y orgánicos (Albaho et al., 2009; Lazcano y Domínguez, 2010). Sin embargo, un sustrato para el cultivo de plantas hortícolas debe reunir ciertas características físicas y químicas que un solo material no las puede cumplir, por lo que es necesario llevar a cabo mezclas de materiales hasta detectar el adecuado balance entre ellas (Cruz-Crespo et al., 2010).

La lombricomposta es un sustrato que se caracteriza por su alto contenido nutrimental, y altas capacidades de retención de agua y de intercambio catiónico (Orozco et al., 1996; Atiyeh et al., 2000; Durán y Henríquez, 2007). Ésta ha remplazado paulatinamente el uso del suelo y de otros sustratos como componentes del medio de crecimiento en la producción hortícola, aunque su uso se recomienda en mezcla con otros materiales (Zaller, 2007; Lazcano y Domínguez, 2010; Ameri et al., 2012). Además, el efecto en el crecimiento de las plantas puede ser diferente entre tipos de lombricomposta debido a la variación en sus propiedades (Doyle et al., 2011). 
En Nayarit, México, se generan diversos subproductos de la actividad agropecuaria e industrial, como: desperdicios de mango provenientes de la industria procesadora de frutas para obtención de concentrados, bagazo de caña de la industria azucarera, y diversos estiércoles, los cuales se han procesado mediante lombricompostaje para así darles un uso útil y reducir o evitar los desechos. También hay minas de tezontle, una roca porosa de origen volcánico que se utiliza como sustrato en los sistemas hidropónicos en la zona centro de México, pero que en Nayarit es escasamente utilizada para ese fin.

Dado el costo de los sustratos importados, como turba ("peat moss") y vermiculita cuyos precios son de $\$ 450$ y $\$ 230$ por $0.1 \mathrm{~m}^{3}$, existe la necesidad de evaluar materiales producidos $\mathrm{u}$ originados localmente. Por otra parte, aunque el cultivo de chile serrano (Capsicum annuum L.) tiene importancia económica, es escasa la información con respecto al uso de soluciones nutritivas y su efecto en la concentración nutrimental del tejido foliar.

El uso de una solución nutritiva determinada repercute en la cantidad total de fertilizantes utilizados, así como en el cuidado de los recursos naturales. Existen diferentes formulaciones de soluciones nutritivas, como la universal de Steiner y la de Hoagland, entre otras. Su uso al $100 \%$ de su concentración depende de diversos factores, como los ambientales, por lo que una forma de proceder es estudiar soluciones nutritivas diluidas en las que se puede regular la cantidad y la proporción relativa de las sales minerales (Gómez-Hernández y Sánchez-del-Castillo, 2003).

Por lo anterior, el objetivo del presente trabajo fue estudiar algunos sustratos (tezontle y mezcla tezontle/lombricomposta) en combinación con varias diluciones de la solución nutritiva de Steiner, y su respectiva interacción, en la concentración nutrimental del tejido foliar y en el crecimiento y rendimiento de chile serrano crecido en condiciones de invernadero.

\section{MATERIALES Y MÉTODOS}

El experimento se estableció en un invernadero ubicado en Xalisco, Nayarit, México, a $21^{\circ} 25^{\prime} 41^{\prime \prime} \mathrm{N}, 104^{\circ} 53^{`} 30^{\prime \prime}$ O y $974 \mathrm{~m}$ de altitud. Las temperaturas mínima y máxima promedios fueron de 24 y $40{ }^{\circ} \mathrm{C}$, respectivamente, con humedad relativa promedio de $80 \%$ y una radiación promedio de $430 \mu \mathrm{mol}$ fotón $\mathrm{m}^{-2} \mathrm{~s}^{-1}$.

El primero de junio de 2011 se sembró semilla de chile serrano variedad 'Tampiqueño' en charolas de poliestireno de 200 cavidades, rellenadas con Sunshine 3 $\AA$ como sustrato, el cual es una mezcla de turba canadiense más vermiculita en proporción 4:1, v/v. Cada charola se regó diariamente con 300 a $820 \mathrm{~mL}$ de la dilución a $25 \%$ de la solución de Steiner (1984), de acuerdo con el crecimiento de la plántula y la condición de clima.

El trasplante a macetas se llevó a cabo a los 55 d después de la siembra, con una plántula por maceta de polietileno negro con capacidad de $15 \mathrm{~L}$ rellena con el sustrato tezontle (T) o la mezcla de tezontle con lombricomposta (T/L) a razón de 4:1, v/v. Las macetas se acomodaron en tres hileras, y cada hilera constó de 12 macetas con el sustrato T, y 12 macetas con el sustrato $\mathrm{T} / \mathrm{L}$, las cuales se distribuyeron al azar a una distancia de $45 \mathrm{~cm}$ entre plantas. La distancia entre hileras fue de $1 \mathrm{~m}$.

El tezontle se obtuvo de una mina de la región y la lombricomposta se formó a partir de hueso de mango-bagazo de caña-estiércol de bovino, en proporción 1:1:1. Las propiedades físicas de los sustratos se muestran en el Cuadro 1 , y las químicas y contenido nutrimental de la lombricomposta y tezontle con lombricomposta se presentan en el Cuadro 2. Estas propiedades se determinaron antes de colocar el material en las macetas.

Después del trasplante se inició la fertilización con tres diluciones de la solución de Steiner: 25, 50 y 75 \%. Así, los tratamientos conformaron un arreglo factorial $3 \times 2$ que

Cuadro 1. Propiedades físicas ${ }^{\dagger}$ de los sustratos.

\begin{tabular}{lccccccc}
\hline Sustrato & $\begin{array}{c}\text { Tamaño de } \\
\text { partícula }(\mathrm{mm})\end{array}$ & PT & PA & CRA (\%) & AFD & AR & ADD \\
\hline $\mathrm{L}$ & 0.5 a 3.3 & 67.0 & 6.6 & 60.5 & 37.0 & 17.0 & 6.5 \\
$\mathrm{~T}$ & 0.18 a 10 & 22.9 & 13.3 & 9.6 & 5.2 & 2.4 & 1.9 \\
$\mathrm{~T} / \mathrm{L}, 4: 1$ & 0.18 a 10 & 46.2 & 6.1 & 40.1 & 4.1 & 4.4 & 31.4 \\
\hline
\end{tabular}

${ }^{\dagger}$ Valor promedio de tres determinaciones. $\mathrm{PT}=$ porosidad total; $\mathrm{PA}=$ porosidad de aire; $\mathrm{CRA}=$ capacidad de retención de agua; $\mathrm{AFD}=$ agua fácilmente disponible; $\mathrm{AR}=$ agua de reserva; $\mathrm{ADD}=$ agua difícilmente disponible; $\mathrm{L}=$ lombricomposta; $\mathrm{T}=$ tezontle; $\mathrm{T} / \mathrm{L}=$ tezontle/ lombricomposta. 
Cuadro 2. Propiedades químicas ${ }^{\dagger}$ y contenido nutrimental de los sustratos.

\begin{tabular}{|c|c|c|c|c|c|c|c|c|c|}
\hline \multirow{2}{*}{ Sustrato } & \multirow[t]{2}{*}{$\mathrm{pH} 1: 4^{\dagger \dagger}$} & \multirow[t]{2}{*}{$\mathrm{CE}\left(\mathrm{dS} \cdot \mathrm{m}^{-1}\right)$} & \multirow[t]{2}{*}{$\mathrm{MO}(\%)$} & \multirow[t]{2}{*}{$\mathrm{CIC}\left(\mathrm{Cmol}_{(+)} \cdot \mathrm{kg}^{-1}\right)$} & $\mathrm{N}$ & $\mathrm{P}$ & $\mathrm{K}$ & $\mathrm{Ca}$ & $\mathrm{Mg}$ \\
\hline & & & & & \multicolumn{5}{|c|}{$(\%)$} \\
\hline $\mathrm{L}$ & 7.3 & 3.9 & 30 & 44.3 & 1.2 & 1.8 & 2.0 & 2.6 & 0.6 \\
\hline $\mathrm{T} / \mathrm{L}, 4: 1$ & 6.0 & 0.3 & - & - & - & - & - & - & - \\
\hline
\end{tabular}

correspondieron a tres diluciones de la solución nutritiva combinadas con dos tipos de sustrato.

En el cálculo del requerimiento de fertilizantes se consideró el análisis de agua: $0.70 \mathrm{Ca}^{+2}, 0.60 \mathrm{Mg}^{+2}, 0.15 \mathrm{~K}^{+}$, $0.10 \mathrm{Na}^{+}, 1.40 \mathrm{HCO}_{3}^{-}, 0.11 \mathrm{SO}_{4}^{-2}, 0.40 \mathrm{Cl}^{-1}$ meq L $\mathrm{L}^{-1}$. Los fertilizantes utilizados fueron $\mathrm{Ca}\left(\mathrm{NO}_{3}\right)_{2} 4 \mathrm{H}_{2} \mathrm{O}$ Yara Liva ${ }^{\circledR}$, $\mathrm{KNO}_{3}$ Ultrasol ${ }^{\circ}, \mathrm{MgSO}_{4} 7 \mathrm{H}_{2} \mathrm{O}$ Sulmag ${ }^{\circledR}, \mathrm{KH}_{2} \mathrm{PO}_{4}$ Peño-

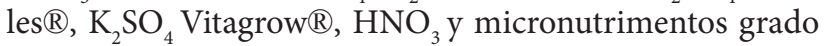
reactivo J. T. Baker ${ }^{\circledR}$, que se utilizaron para preparar la solución madre con: $2.8 \mathrm{~g} \mathrm{~L}^{-1}$ de $\mathrm{H}_{3} \mathrm{BO}_{3}, 2.2 \mathrm{~g} \mathrm{~L}^{-1}$ de $\mathrm{MnSO}_{4}$ $\mathrm{H}_{2} \mathrm{O}, 0.4 \mathrm{~g} \mathrm{~L}^{-1}$ de $\mathrm{ZnSO}_{4}, 0.08 \mathrm{~g} \mathrm{~L}^{-1}$ de $\mathrm{CuSO}_{4}, 0.1 \mathrm{~g} \mathrm{~L}^{-1}$ de Na${ }_{2} \mathrm{MoO}_{4}$ y $3 \mathrm{mg} \mathrm{L}^{-1}$ de Fe-EDTA. De esta solución se tomaron alícuotas de $1 \mathrm{~mL}$ para agregar a $1.5,2.0$ y $2.5 \mathrm{~L}$ de agua y así formar las diluciones a 25,50 y $75 \%$, respectivamente. El $\mathrm{pH}$ de las diluciones se ajustó a 5.5 y la conductividad eléctrica fue de $0.56,1.20$ y $1.71 \mathrm{dS} \mathrm{m}^{-1}$ para las concentraciones respectivas antes mencionadas.

Se aplicaron $250 \mathrm{~mL}$ por planta en cada riego por goteo, y de dos a seis riegos por día, en función de la etapa fenológica y la condición de clima. Para la distribución de las diluciones de acuerdo con los tratamientos, se colocaron tres líneas de riego por cada tanque con la dilución nutritiva correspondiente, por lo que cada hilera de macetas contó con tres líneas de riego. El control de mosquita blanca (Bemisia tabaci (Gennadius)) se hizo mediante la colocación de trampas y aplicación del insecticida Imidacloprid ${ }^{\circledR}$ a una dosis de $1 \mathrm{~mL} \mathrm{~L}^{-1}$ de agua.

Como variables de respuesta se midió: diámetro de tallo de cada planta, con vernier digital Trupper ${ }^{\circledR}$ modelo CALDI-6MP (Estado de México, México) a $10 \mathrm{~cm}$ arriba del nivel del sustrato; número de ramificaciones por planta; biomasa seca de la planta, para lo cual se cortó a nivel de sustrato y se secó a $70^{\circ} \mathrm{C}$ por $72 \mathrm{~h}$ en estufa con circulación de aire Blue $M{ }^{\circledR}$ modelo SW-17TA (Illinois, USA) y después se pesó. A la cosecha de fruto, que se hizo cuando presentó una coloración verde brillante, se pesó con una balanza Precisa ${ }^{\circledR}$ modelo BJ 1200C (Berna, Suiza) para obtener el rendimiento total de fruto por planta durante cuatro cortes. Todas las variables, con excepción del rendimiento, se determinaron a los 40 y $80 \mathrm{~d}$ después del trasplante (ddt).
Se determinó la concentración nutrimental en las hojas recientemente maduras en la parte superior de la planta. Las muestras de hoja se sometieron a digestión húmeda y en el extracto obtenido se determinó $\mathrm{N}$ total por el método micro Kjeldahl (Alcántar-González y Sandoval-Villa, 1999). P se midió con el método de amarillo vanadato molibdato mediante un espectrofotómetro UV-Visible Thermo Fisher Scientific modelo Genesys ${ }^{\mathrm{TM}} 20{ }^{\circledR}$ (Wisconsin, USA). Ca y $\mathrm{Mg}$ se determinaron en un espectrofotómetro Varian modelo SpectrAA-50® (Canberra, Australia). K se determinó en un flamómetro Sherwood modelo $410 \AA$ (Cambrige, U.K.).

Los datos de las variables se analizaron en un diseño completamente al azar, con arreglo factorial $3 \times 2$ con ocho repeticiones, y se aplicó la prueba de comparación de medias de Tukey $(\mathrm{P} \leq 0.05)$ con el programa SAS (SAS Institute, 1999). La unidad experimental consistió de una maceta con una planta.

\section{RESULTADOS Y DISCUSIÓN}

\section{Interacción dilución de la solución nutritiva $\mathrm{x}$ sustrato}

A los 40 ddt la interacción dilución de la solución nutritiva $\mathrm{x}$ sustrato contribuyó en la variable diámetro de tallo en con $53 \%$ a la suma de cuadrados de tratamientos, mientras que $4 \%$ de dicha variación se debió al efecto de la dilución . Para la variable biomasa seca de planta medida a los $80 \mathrm{ddt}$, la interacción dilución x sustrato contribuyó con $9 \%$ a la suma de cuadrados de tratamientos, en tanto que el efecto de la dilución coadyuvó en $84 \%$, y el efecto sustrato con 6 $\%$. Las demás variables evaluadas se explicaron sólo en función de cada factor estudiado, ya que la interacción dilución $\mathrm{x}$ sustrato no fue significativa.

\section{Efecto de la dilución de la solución nutritiva en crecimiento y rendimiento de fruto}

Las dosis de la dilución nutritiva difirieron en su efecto en todas las variables de crecimiento excepto en el número de ramificaciones por planta a los $40 \mathrm{ddt}$ (Cuadro 3). A los 80 ddt los valores de diámetro de tallo, número de ramificaciones y biomasa seca de planta fueron mayores en los 
Cuadro 3. Variables de crecimiento de chile serrano var. 'Tampiqueño' en invernadero.

\begin{tabular}{|c|c|c|c|c|c|c|c|}
\hline \multirow{2}{*}{ Factor } & $\mathrm{DT}(\mathrm{cm})$ & NR & BSP (g/planta) & $\mathrm{DT}(\mathrm{cm})$ & NR & BSP (g/planta) & \multirow{2}{*}{$\begin{array}{l}\text { Rendimiento } \\
\text { total (g/planta) }\end{array}$} \\
\hline & & $40 \mathrm{ddt}$ & & \multicolumn{3}{|c|}{$80 \mathrm{ddt}$} & \\
\hline \multicolumn{8}{|c|}{ Solución (SO) } \\
\hline 75 & $0.5 \mathrm{~b}^{\dagger}$ & $6.3 \mathrm{a}$ & $7.4 \mathrm{a}$ & $1.6 \mathrm{a}$ & $9.0 \mathrm{a}$ & $211.0 \mathrm{a}$ & $1517.8 \mathrm{a}$ \\
\hline 50 & $0.6 \mathrm{a}$ & $6.0 \mathrm{a}$ & $6.1 \mathrm{~b}$ & $1.6 \mathrm{a}$ & $8.1 \mathrm{a}$ & $194.0 \mathrm{a}$ & $1341.9 \mathrm{~b}$ \\
\hline 25 & $0.5 \mathrm{c}$ & $6.0 \mathrm{a}$ & $4.8 \mathrm{c}$ & $1.2 \mathrm{~b}$ & $5.3 \mathrm{~b}$ & $126.0 \mathrm{~b}$ & $884.4 \mathrm{c}$ \\
\hline \multicolumn{8}{|c|}{ Sustrato (SU) } \\
\hline $\mathrm{T} / \mathrm{L}$ & $0.54 \mathrm{a}$ & $6.8 \mathrm{a}$ & $6.6 \mathrm{a}$ & $1.5 \mathrm{a}$ & $8.7 \mathrm{a}$ & $187 \mathrm{a}$ & $1221.0 \mathrm{a}$ \\
\hline $\mathrm{T}$ & $0.54 \mathrm{a}$ & $5.4 \mathrm{~b}$ & $5.6 \mathrm{~b}$ & $1.4 \mathrm{~b}$ & $6.2 \mathrm{~b}$ & $168 \mathrm{~b}$ & $1274.6 \mathrm{a}$ \\
\hline $\mathrm{SO} \times \mathrm{SU}$ & * & ns & ns & ns & ns & * & ns \\
\hline
\end{tabular}

${ }^{\dagger}$ Medias con letras iguales en una columna para cada factor, no son estadísticamente diferentes (Tukey, 0.05 ); ns = no significativo; ${ }^{*}=$ significativo con $\mathrm{P} \leq$ 0.05; $\mathrm{DT}=$ diámetro de tallo; $\mathrm{NR}=$ número de ramificaciones; $\mathrm{BSP}=$ biomasa seca de planta; $\mathrm{ddt}=$ días después del trasplante $; \mathrm{L}=$ lombricomposta; $\mathrm{T} / \mathrm{L}=$ tezontle/lombricomposta.

tratamientos con las diluciones a 50 y $75 \%$, con respecto de la dilución a $25 \%$ de fuerza iónica. El rendimiento de fruto fue significativamente mayor en aproximadamente 12 y 41 $\%$ en las plantas tratadas con dilución a $75 \%$, con respecto del obtenido con 50 y $25 \%$. Estas diferencias se atribuyen a que en la dilución de $75 \%$ los nutrimentos estuvieron en mayor disponibilidad para la planta de chile durante la etapa de fructificación cuando hay mayor demanda de nutrimentos (Azofeifa y Moreira, 2005).

En tomate (Solanum lycopepersicum L.), Cruz-Crespo et al. (2012) no encontraron diferencia significativa entre las diluciones nutritivas a 50 y $75 \%$ para diámetro de tallo, biomasa seca de planta y rendimiento de fruto, mientras que en chile pimiento (Capsicum annuum L.) Villa-Castorena et al. (2009) encontraron que las diluciones a 75 y $90 \%$ fueron similares entre sí pero con mayor rendimiento de fruto que la dilución a 60 \%. En cambio, Valentín-Miguel et al. (2013) mostraron que para chile de agua (Capsicum annuum L.) hubo incremento significativo de biomasa seca de planta y rendimiento de fruto al aumentar la concentración de las diluciones de 25, 50 y $75 \%$, situación similar a la que se observó en este estudio con chile serrano.

Es importante señalar que la solución de Steiner a concentración de $100 \%$ no fue evaluada, por lo que en estudios posteriores se recomienda considerarla, ya que la magnitud del rendimiento incrementó significativamente al aumentar su concentración de 25 a $75 \%$. No obstante, con base en los resultados obtenidos de biomasa seca de planta y rendimiento de fruto se propone aplicar la dilución nutritiva a 75 $\%$ desde el momento del trasplante.

\section{Efecto del sustrato en crecimiento y de rendimiento de fruto}

Los sustratos utilizados también afectaron a las variables de crecimiento número de ramificaciones y biomasa seca de planta, aunque en el rendimiento de fruto no se manifestó tal efecto (Cuadro 3). Con el sustrato T/L se logró mayor número de ramificaciones y mayor biomasa seca de planta tanto a los 40 como a los $80 \mathrm{ddt}$, con respecto al sustrato T, superioridad que fue de 21 y $19 \%$ para número de ramificaciones y de 14 y $11 \%$ para biomasa seca de planta, respectivamente. En tanto que el diámetro de tallo fue mayor sólo a los $80 \mathrm{ddt}$.

En cuanto al rendimiento de fruto los resultados difieren con los obtenidos por Arancon et al. (2005) quienes reportaron un mayor incremento de biomasa seca, crecimiento y rendimiento de fruto en chile pimiento cultivado en suelo abonado con lombricomposta de estiércol, lo que atribuyeron no sólo al contenido nutrimental de la lombricomposta sino también al incremento de la actividad y biomasa microbiana. Tales autores señalan que esto pudo deberse al aumento de la producción de reguladores de crecimiento como ácidos húmicos y hormonas. Por su parte, Oliva-Llaven et al. (2008) encontraron mayor diámetro de tallo del chile pimiento en un sustrato de lombricomposta más suelo $(1: 3, v / v)$, en relación al testigo donde sólo se utilizó suelo.

\section{Efecto de la dilución de la solución nutritiva en la concentración nutrimental foliar}

En cuanto a la concentración de nutrimentos, las plantas regadas con la dilución a $75 \%$ siempre presentaron mayor concentración de nitrógeno foliar en comparación con el resto de las diluciones, aunque sin diferencia respecto a 50 $\%$ a los 80 ddt (Cuadros 4 y 5). Para P sólo se encontró diferencia a los 40 ddt (Cuadro 4), donde la dilución a $75 \%$ superó a las diluciones de 25 y 50 \%. Para el caso de K las plantas presentaron la misma concentración nutrimental entre diluciones, en ambas fechas de muestreo. En el Ca se encontró diferencia en ambas fechas de muestreo, donde 
Cuadro 4. Concentración de macronutrimentos en hojas de chile serrano var. 'Tampiqueño' crecido en invernadero, a los $40 \mathrm{~d}$ después del trasplante.

\begin{tabular}{|c|c|c|c|c|c|}
\hline \multirow{2}{*}{ Factor } & $\mathrm{N}$ & $\mathrm{P}$ & $\mathrm{K}$ & $\mathrm{Ca}$ & $\mathrm{Mg}$ \\
\hline & \multicolumn{5}{|c|}{$\left(\mathrm{g} \mathrm{kg}^{-1}\right)$} \\
\hline \multicolumn{6}{|c|}{ Solución (SO) } \\
\hline 75 & $51.6 \mathrm{a}^{\dagger}$ & $5.3 \mathrm{a}$ & $62.8 \mathrm{a}$ & $29.9 \mathrm{a}$ & $6.9 \mathrm{a}$ \\
\hline 50 & $47.5 \mathrm{~b}$ & $4.7 \mathrm{~b}$ & $65.7 \mathrm{a}$ & $28.6 \mathrm{a}$ & $6.6 \mathrm{a}$ \\
\hline 25 & $45.3 \mathrm{~b}$ & $4.6 \mathrm{~b}$ & $61.9 \mathrm{a}$ & $24.1 \mathrm{~b}$ & $7.2 \mathrm{a}$ \\
\hline \multicolumn{6}{|c|}{ Sustrato (SU) } \\
\hline $\mathrm{T} / \mathrm{L}$ & $48.3 \mathrm{a}$ & $5.1 \mathrm{a}$ & $64.4 \mathrm{a}$ & $29.0 \mathrm{a}$ & $7.3 \mathrm{a}$ \\
\hline $\mathrm{T}$ & $48.0 \mathrm{a}$ & $4.6 \mathrm{~b}$ & $62.5 \mathrm{a}$ & $26.1 \mathrm{~b}$ & $6.5 \mathrm{a}$ \\
\hline $\mathrm{SO} \times \mathrm{SU}$ & ns & ns & ns & ns & ns \\
\hline
\end{tabular}

Cuadro 5. Concentración de macronutrimentos en hojas de chile serrano var. 'Tampiqueño' crecido en invernadero, a los $80 \mathrm{~d}$ después del trasplante.

\begin{tabular}{|c|c|c|c|c|c|}
\hline \multirow{2}{*}{ Factor } & $\mathrm{N}$ & $\mathrm{P}$ & $\mathrm{K}$ & $\mathrm{Ca}$ & $\mathrm{Mg}$ \\
\hline & \multicolumn{5}{|c|}{$\left(\mathrm{g} \mathrm{kg}^{-1}\right)$} \\
\hline \multicolumn{6}{|c|}{ Solución (SO) } \\
\hline 75 & $45.7 \mathrm{a}^{\dagger}$ & $2.4 \mathrm{a}$ & $48.6 \mathrm{a}$ & $67.6 \mathrm{a}$ & $12.4 \mathrm{a}$ \\
\hline 50 & $43.0 \mathrm{a}$ & $2.4 \mathrm{a}$ & $50.8 \mathrm{a}$ & $60.3 \mathrm{ab}$ & $11.8 \mathrm{ab}$ \\
\hline 25 & $37.8 \mathrm{~b}$ & $2.5 \mathrm{a}$ & $48.2 \mathrm{a}$ & $50.5 \mathrm{~b}$ & $11.4 \mathrm{~b}$ \\
\hline \multicolumn{6}{|c|}{ Sustrato (SU) } \\
\hline $\mathrm{T} / \mathrm{L}$ & $43.4 \mathrm{a}$ & $2.6 \mathrm{a}$ & $50.8 \mathrm{a}$ & $62.6 \mathrm{a}$ & $12.8 \mathrm{a}$ \\
\hline $\mathrm{T}$ & $40.9 \mathrm{a}$ & $2.3 \mathrm{~b}$ & $47.6 \mathrm{a}$ & $56.4 \mathrm{a}$ & $11.0 \mathrm{~b}$ \\
\hline SO x SU & ns & ns & ns & ns & ns \\
\hline
\end{tabular}

las diluciones a 50 y $75 \%$ fueron superiores que la de 25 $\%$. En contenido foliar de $\mathrm{Mg}$, únicamente a los $80 \mathrm{ddt}$ las concentraciones a 50 y $75 \%$ resultaron las de mayor valor.

El incremento en concentración foliar de algunos nutrimentos por efecto de la dilución nutritiva aportada, se debió a la mayor disponibilidad nutrimental para abastecer la demanda de chile serrano durante el crecimiento de la planta, como lo explicaron Gorbe y Calatayud (2010). En relación a esto, a los $40 \mathrm{ddt}$ las variables biomasa seca de planta y el número de ramificaciones mostraron correlación positiva significativa de 0.73 y 0.60 con la concentración de Ca, respectivamente. A los 80 ddt la biomasa seca de planta presentó correlación positiva significativa de 0.51 con $\mathrm{N}$ y Ca, y de 0.52 del rendimiento con $\mathrm{N}$ y Ca.

Se observó que las concentraciones de N, P y K disminuyeron con la edad de la planta, en tanto que las de Ca y Mg se incrementaron (Cuadros 4 y 5), lo cual coincide con las tendencias generales observadas en tejidos vegetales durante el ciclo de un cultivo (Marschner, 2012), observado también en pimiento y tomate (Ojodeagua-Arredondo et al., 2008; Noh-Medina et al., 2010). No se encontró reporte alguno que informe la concentración nutrimental del chile serrano, pero los valores a los $80 \mathrm{ddt}$ se ubicaron dentro de las concentraciones mínima y máxima en hojas de chile pimiento reportadas por Terbe et al. (2006).

Al considerar lo reportado por Mills y Benton (1996) para chile pimiento, $\mathrm{N}, \mathrm{K}$ y Mg se encontraron dentro del rango de suficiencia, el cual es 4 a $6 \% \mathrm{~N}$; 3 a $6 \% \mathrm{~K}$; 0.3 a $1 \% \mathrm{Mg}$; pero el $\mathrm{P}$ se ubicó abajo del límite inferior del rango de suficiencia, el cual comprende de 0.35 a $1 \%$ de $\mathrm{P}$; Ca se situó fuera del límite superior, cuyo rango de suficiencia es entre 1.30 a $2.8 \%$. Por ello se considera que las diluciones nutritivas utilizadas no fueron suficientes en la 
concentración de $\mathrm{P}$, mientras que para Ca fue excesiva.

\section{Efecto del sustrato en la concentración nutrimental foliar}

Se encontró efecto del sustrato en la concentración foliar de P, Ca y Mg. A los $40 \mathrm{ddt}$ el sustrato T/L obtuvo un incremento de 9 y $10 \%$ para $\mathrm{P}$ y Ca, respectivamente, en comparación con el sustrato $\mathrm{T}$ (Cuadro 4). En cambio, a los 80 ddt el sustrato T/L superó al T en $13 \%$ para $\mathrm{P}$ y en $14 \%$ para Mg (Cuadro 5).

Los materiales de lombricomposta usualmente contienen elementos minerales que son más fácilmente disponibles para las plantas (Evanylo et al., 2008; Hernández et al., 2010), lo que explica el incremento de los contenidos foliares de P, Ca y Mg. Arancon et al. (2005) reportaron que la concentración de $\mathrm{P}$ fue mayor en el tejido foliar de chile pimiento cultivado con diferentes lombricompostas y fertilización química complementaria, que cuando fue crecido sólo con fertilizantes inorgánicos. Jouquet et al. (2011) reportaron que el N, P y K provenientes de la fertilización química, se lixiviaron en menor cantidad en el suelo cuando se incorporó lombricomposta, lo que se debió en parte a la lenta tasa de mineralización y a la más alta capacidad de intercambio catiónico.

La concentración similar de $\mathrm{N}, \mathrm{K}$ y Ca en el tejido foliar de las plantas de ambos sustratos (Cuadro 5), quizás explica la obtención de iguales rendimientos, cuando se esperaba mayor rendimiento en el sustrato T/L (Cuadro 3). Al respecto, Arancon et al. (2004) afirmaron que además de la concentración nutrimental hay que considerar las propiedades físicas de los sustratos, lo cual en diversos trabajos no se tiene en cuenta.

En el Cuadro 1 se muestra que el T presentó menor porosidad total y capacidad de retención de agua, con lo que se esperaría una disminución en el crecimiento y rendimiento, como lo indicaronn Ortega-Martínez et al. (2010). Sin embargo, el sustrato T manifestó mayor porosidad de aire e igual valor de agua fácilmente disponible respecto del sustrato T/L. Benito et al. (2005) señalaron que la producción de pasto en una composta de residuos de jardinería fue de $0.61 \mathrm{~g} /$ maceta, mientras que en una mezcla de compost con turba $(9: 1, \mathrm{v} / \mathrm{v})$ fue de $0.77 \mathrm{~g} /$ maceta, y que en la mezcla (3:1, $\mathrm{v} / \mathrm{v})$ el rendimiento fue de $0.76 \mathrm{~g} /$ maceta, mezclas en las que los valores de porosidad total, porosidad de aire y agua fácilmente disponible fueron: $88.7,89,89.4 \%$; 48.4, 42.6, $38.3 \%$ y $9.2,12.9,14.8 \%$, respectivamente, y se observó que tanto la porosidad de aire y agua fácilmente disponible influyeron en el resultado obtenido.

La porosidad total es una de las propiedades más impor- tantes para el crecimiento y desarrollo de los cultivos ( $\mathrm{Ca}$ brera, 1999; Ortega-Martínez et al., 2010). Según Ansorena-Miner (1994), la porosidad de aire es la propiedad física más importante de los sustratos. Al respecto, Morard et al. (2000), Tesi et al. (2003) y Urrestarazu y Mazuela (2005) indicaron que la deficiencia de oxígeno para la respiración de la raíz puede ser un factor limitante, por tener efecto inmediato en la absorción de agua y de nutrimentos, lo que afecta de manera negativa al rendimiento.

El tezontle aquí utilizado tuvo menos agua fácilmente disponible de la mezcla $\mathrm{T} / \mathrm{L}$, lo que posiblemente repercutió en el rendimiento (Cuadro 1 y 3). Márquez-Hernández et al. (2008) reportaron que el rendimiento de tomate fue menor en una mezcla de lombricomposta con arena, en comparación a la mezcla de lombricomposta con perlita en la misma proporción. Por los resultados aquí obtenidos se recomienda no utilizar la mezcla $\mathrm{T} / \mathrm{L}(4: 1, \mathrm{v} / \mathrm{v})$, dado que el rendimiento fue igual al sustrato $\mathrm{T}$, además de que el tezontle provino de una mina de la región de Nayarit donde los materiales pueden ser variables. Por esto se sugiere estudiar la lombricomposta con otro tipo de tezontle o con otros materiales minerales u orgánicos de la región.

\section{CONCLUSIONES}

Las plantas de chile serrano regadas con la dilución a 75 $\%$ de la solución nutritiva de Steiner expresaron mayor crecimiento y mayor concentración nutrimental de nitrógeno, calcio y magnesio en tejido foliar, y produjeron $41 \%$ más rendimiento de fruto que el obtenido en las plantas con la dilución a $25 \%$. Los valores de la concentración nutrimental de chile serrano por efecto de la dilución a $75 \%$ se ubicaron en el rango de suficiencia, con excepción del fósforo y calcio, por lo que la dilución a $75 \%$ fue insuficiente para fósforo y sobredosificada en calcio.

El sustrato tezontle/lombricomposta (4:1, v/v) favoreció el crecimiento de la planta de chile serrano y la concentración nutrimental de fósforo y magnesio en el tejido foliar, más no tuvo efecto en el rendimiento de fruto en comparación con el sustrato tezontle, por lo que se considera no apto para la producción.

\section{BIBLIOGRAFÍA}

Albaho M., N. Bhat, H. A. Rezq and B. Thomas (2009) Effect of three different substrates on growth and yield of two cultivars of Capsicum annuum. European Journal of Scientific Research 28:227-233.

Alcántar-González G. y M. Sandoval-Villa (1999) Manual de Análisis Químico de Tejido Vegetal. Guía de Muestreo, Preparación, Análisis e Interpretación. Publicación especial 10. Sociedad Mexicana de la Ciencia del Suelo. Chapingo, México. 155 p.

Ameri A., A. Tehranifar, M. Shoor and G. H. Davarynejad (2012) Effect of substrate and cultivar on growth characteristic of strawberry in soilless culture system. African Journal of Biotechnology 
11:11960-11966

Ansorena-Miner J. (1994) Sustratos. Propiedades y Caracterización. Ed. Mundi-Prensa. Madrid, España. 172 p.

Arancon N. Q., A. E. Clive, R. Atiyeh and J. D. Metzger (2004) Effects of vermicomposts produced from food waste on the growth and yields of greenhouse peppers. Bioresource Technology 93:139144

Arancon N. Q., C. A. Edwards, P. Biermanb, J. D. Metzger and C. Luchtd (2005) Effects of vermicomposts produced from cattle manure, food waste and paper waste on the growth and yield of peppers in the field. Pedobiologia 49:297-306.

Atiyeh R. M., S. Subler, C. A. Edwards, G. Bachman, J. D. Metzger and W. Shuster (2000) Effects of vermicomposts and composts on plant growth in horticultural container media and soil. Pedobiologia 44:579-590.

Azofeifa A. y M. A. Moreira (2005) Absorción y distribución de nutrimentos en plantas de chile dulce (Capsicum annuum CV. UCR 589) en Alajuela, Costa Rica. Agronomía Costarricence 29:77-84

Benito M., A. Masaguer, R. De-Antonio and A. Moliner (2005) Use of pruning waste compost as a component in soilless growing media. Bioresource Technology 96:597-603.

Cabrera I. R. (1999) Propiedades, uso y manejo de sustratos de cultivo para la producción de plantas en maceta. Revista Chapingo Se rie Horticultura 5:5-11.

Cruz-Crespo E., M. Sandoval-Villa, V. H. Volke-Haller, A. Can-Chulim y J. Sánchez-Escudero (2012) Efecto de mezclas de sustratos y concentración de la solución nutritiva en el crecimiento y rendimiento de tomate. Revista Mexicana de Ciencias Agrícolas 3:1361-1373.

Cruz-Crespo E., M. Sandoval-Villa, V. Volke-Haller, V. Ordaz-Chaparro, J. L. Tirado-Torres y J. Sánchez Escudero (2010) Generación de mezclas de sustratos mediante un programa de optimización utilizando variables físicas y químicas. Terra Latinoamericana 28:219-229.

Doyle O. P. E., C. King, R. O'Haire, T. Moore, D. NiChualain and W. Carlile (2011) The effect of a peat based growing medium, with varying fertilizer levels and amended with composted green waste (CGW), on the growth and development of tomato (Lycopersicon lycopersicum (L.). Acta Horticulturae 891:93:102.

Durán L. y C. Henríquez (2007) Caracterización química, física y microbiológica de vermicompostes producidos a partir de cinco sustratos orgánicos. Agronomía Costarricense 31:41-51.

Evanylo G., C. Sherony, J. Spargo, D. Starner, M. Brosius and K. Haering (2008) Soil and water environmental effects of fertilizermanure-and compost-based fertility practices in an organic vegetable cropping system. Agriculture, Ecosystems and Environment 127:50-58.

Gómez-Hernández T. y F. Sánchez-del-Castillo (2003) Soluciones nutritivas diluidas para la producción de jitomate a un racimo. Terra Latinoamericana 21:57-63.

Gorbe E. and A. Calatayud (2010) Optimization of nutrition in soilless systems: a review. Advances in Botanical Research 53:193-245.

Hernández A., H. Castillo, D. Ojeda, A. Arras, J. López and E. Sánchez (2010) Effect of compost on lettuce production. Chilean Journal of Agricultural Research 70:583-589.

Jouquet E. P., E. Bloquel, T. Thu-Doan, M. Ricoy, D. Orange, C. Rumpel and T. Tran-Duc (2011) Do compost and vermicompost improve macronutrient retention and plant growth in degraded tropical soils? Compost Science \& Utilization 19:15-24.

Lazcano C. and J. Domínguez (2010) Effects of vermicompost as a potting amendment of two commercially-grown ornamental plant species. Spanish Journal of Agricultural Research 8:1260-1270.
Márquez-Hernández C., P. Cano-Ríos y N. Rodríguez-Dimas (2008) Uso de sustratos orgánicos para la producción de tomate en invernadero. Agricultura Técnica en México 34:69-74.

Marschner P. (2012) Marschner's Mineral Nutrition of Higher Plants. $3^{\text {rd }}$ ed. Academic Press. London. 649 p.

Mills H. A. and J. Benton (1996) Plant Analysis Handbook II. A Practical Sampling, Preparation, Analysis and Interpretation Guide. Ed. Micro Macropublishing. Georgia, USA. 422 p.

Morard P., L. Lacoste and J. Silvestre (2000) Effect of oxygen deficiency on uptake of water and mineral nutrients by tomato plants in soilless culture. Journal of Plant Nutrition 23:1063-1078.

Noh-Medina J., L. Borges-Gómez y M. Soria-Fregoso (2010) Composición nutrimental de biomasa y tejidos conductores en chile habanero (Capsicum chinense Jacq.). Tropical and Subtropical Agroecosystems 12:219-228

Ojodeagua-Arredondo J. L., J. Z. Castellanos-Ramos, J. J. Muñoz-Ramos, G. Alcántar-González, L. Tijerina-Chávez, P. VargasTapia y S. Enríquez-Reyes (2008) Eficiencia de suelo y tezontle en sistemas de producción de tomate en invernadero. Revista Fitotecnia Mexicana 31:367-374.

Oliva-Llaven M. A., J. L. Guzman-Jiménez, B. I. Cabrera-Coro, R. Rincón-Rosales, J. Montes-Molina, L Dendooven and F. A. Gutiérrez-Miceli (2008) Fruit characteristics of bell pepper cultivated in sheep manure vermicompost substituted soil. Journal of Plant Nutrition 31:1585-1598.

Orozco F. H., J. Cegarra, L. M. Trujillo and A. Roig (1996) Vermicomposting of coffee pulp using the earthworm Eisenia fétida: effects on $\mathrm{C}$ and $\mathrm{N}$ contents and the availability of nutrient. Biology and Fertility of Soil 22:162-166.

Ortega-Martínez L. D., J. Sánchez-Olarte, R. Díaz-Ruiz y J. OcampoMendoza (2010) Efecto de diferentes sustratos en el crecimiento de plántulas de tomate (Lycopersicum esculentum Mill). $R a$ Ximhai 6:365-372.

SAS Institute (1999) Statistical Analysis System (SAS) Versión 8 User's guide. Cary, N. C. USA. 584 p.

Steiner A. A. (1984) The Universal Nutrient Solution. Proceeding Sixth International Congress on Soilless Culture. Wageningen. The Netherlands. pp:633-650.

Terbe I., Z. Szabó and N. Kappel (2006) Macronutrient accumulation in green pepper (Capsicum annuum L.) as affected by different production technologies. International Journal of Horticultural Science 12:13-19.

Tesi R., A. Lenzi and P. Lombardi (2003) Effect of salinity and oxygen level on lettuce grown in a loading system. Acta Horticulturae 609:383-387.

Urrestarazu M. and P. C. Mazuela (2005) Effect of slow-release oxygen supply by fertigation on horticultural crops under soilless culture. Scientia Horticulturae 106:484-490.

Valentín-Miguel M. C., R. Castro-Brindis, J. E. Rodríguez-Pérez y M. Pérez-Grajales (2013) Extracción de macronutrimentos en chile de agua (Capsicum annuum L.). Revista Chapingo Serie Horticultura 19:71-78.

Villa-Castorena M., E. A. Catalán-Valencia, M. A. Insunza-Ibarra, A. Román-López, M. L. González-López y J. Valdéz-Amaya (2009) Cultivares y nutrición de chile pimiento (Capsicumannuum L.) en invernadero de clima controlado. Biotecnia 11:45 49.

Zaller J. G. (2007) Vermicompost as a substitute for peat in potting media: Effects on germination, biomass allocation, yields and fruit quality of three tomato varieties. Scientia Horticulturae 112:191-199. 